\title{
Influence of Boundary Conditions on Ceramic/Metal Plates under Ballistic Loads
}

\author{
Nadda Jongpairojcosit \\ Defence Technology Institute (Public Organisation), Changwattana, Thailand \\ Email: nadda.j@dti.or.th
}

Received 27 March 2015; accepted 24 June 2015; published 1 July 2015

\begin{abstract}
Ceramic/metal plate is one of the most widely used light weight armors, especially to protect armor piercing (AP) bullet. Experimental investigation of projectile penetration mechanism into the ceramic/metal plate requires costly sensitive equipment to capture impact phenomenon that completes within microseconds. Alternatively, the impact mechanism can be efficiently investigated using numerical simulations. Among recent investigations on the protective capability of this ceramic/metal plates, few only discussed the influence of the boundary effects on the ballistic protection. This study thus aims to examine the effect of boundary conditions by changing shapes of the plate, border constraints and bounded materials in numerical simulation. Material models of the ceramic and the backing metal plate made of aluminium 2017-T6 are selected. The 7.62 AP projectile's core was modeled by a solid cylinder. The initial projectile velocity was $940 \mathrm{~m} / \mathrm{s}$. The plates are represented by either a square or a hexagonal tile. The edges of the plates were fixed or enclosed by a soft epoxy. To investigate the effect of backing plate, a small gap was introduced between some of the ceramic and aluminum interfaces. The results showed that the hexagonal tiles reduce the deformation of the backing plate. The plates bounded by the epoxy exhibit inferior performances compared to the fixed plates. Finally, the small gap between the ceramic and the aluminum interfaces significantly increases the time to stop the projectile.
\end{abstract}

\section{Keywords}

Ceramic Armor, Boundary Condition, Impact Behavior

\section{Introduction}

One classical armor material is monolithic steel i.e. Rolled Homogeneous Armor (RHA). This material disperses the impact energy from projectile through its plastic deformation. The limitations of steel armors are their high density, maximum deformation and low protection against Armor-piercing (AP) bullet. For these reasons, composite armors have been developed. Ceramic/metal is one of the widely used composite armor materials. Ceramic/metal armor has less areal density compared with the monolithic steel. As a result of increasing capability of payload and vehicle maneuverability, the armor vehicle performance is improved. Besides, the hardness of ceramic face help to erode the AP projectile head, reduce its stability and slow down the projectile speed. The penetration efficiency of the blunted AP projectile was also reduced because the energy disperses to the backing 
plate on larger area. With the same amount of energy, plastic deformation of backing plate hit by blunted projectile has small depth compared with that of the sharp one which decreases damages behind the backing plate.

Failure of ceramic tile under the high velocity impact is caused by the stress wave in the material. When the projectile hits the ceramic face, the shock wave, which is compressive wave, will transfer from the ceramic front surface to its back. At the connecting surface between the ceramic and the backing plate, some of the shock wave will continue moving into the steel and the remaining shock wave will reflect back to the ceramic. This wave reflection creates tensile wave in the material. Since brittle materials have low tensile strength, the ceramic starts to crack under the tensile wave.

The crack from this impact has conical shape which is called conoid. The dimensions of the conoid have been widely studied because the radius of the cone base has an effect on the energy dispersion from the ceramic to the backing plate.

The ballistic performance of the ceramic protection depends on various parameters. P.J. Hazell et al. [1] found that increasing the density of the ceramic compact for given thickness yields better result in ballistic resistance than increasing the thickness of a given density.

The thickness and properties of adhesive layer in ceramic/metal armor also influences the ballistic protection [2] [3]. Paul and Colin et al. [4] have shown the optimum design of ceramic tile size on ballistic performance. However, there are a limited number of publications on the boundary effects on numerical ballistic simulation. This work shows the influences of ceramic tiles' boundary conditions on ballistic performance using numerical simulation.

\section{Impact Mechanics}

\subsection{Ceramic Fracture}

Ceramic fracture under impact has different physical characteristic compare to other armor steel materials because of its brittleness. Ceramic has high compressive strength but low tensile strength. For these reasons, it can resist the compressive shock wave after the impact. On the other hand, it starts to crack because of the tensile shock wave generated by rarefaction wave. When the stress is higher than its strength limit, the tile is broken into pieces known as spalling. To prevent the danger from the spalling, ceramic armor has to be supported by backing sheet such as steel and fibers.

The fracture of ceramic armor with the backing plate is different from the characteristics previously described.

One important behavior is conical fracture or the conoid. The interest of the conoid is that it increases the area transferring kinetic energy from the projectile to the backing plate. For a larger area, the impact energy can be better distributed to the rear plate as shown in Figure 1. In general, the time that fracture conoid developed can be calculated by Equation (1) [5]. However, it is essential to note that this equation does not take into account the material properties of ceramic, velocity of impact and the tiles' boundary conditions.

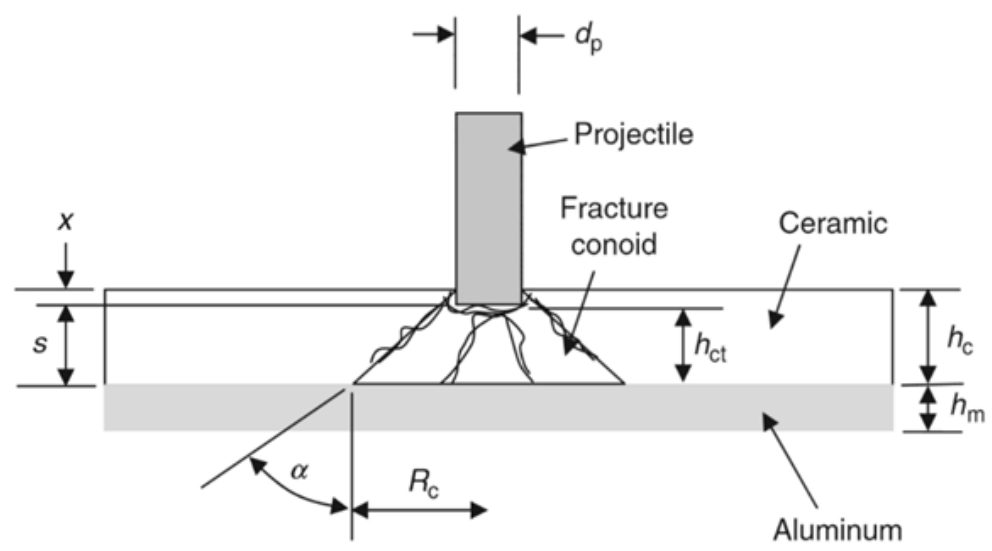

Figure 1. Fracture conoid in ceramic material (Model of Zaera and SanchezGalvez) [6]. 


$$
t_{\text {conoid }}=\frac{h_{c}}{C_{L}}+\frac{h_{c}}{V_{\text {rad,crack }}}
$$

where $h_{c}$ represents the thickness of the ceramic as illustrated in Figure 1. $C_{L}$ is the longitudinal wave speed in the material and $V_{\text {rad,crack }}$ is the speed of radial crack growth.

\subsection{Numerical Model}

To investigate the impact mechanism, non-linear dynamic simulation with high-deformation and short-duration has to be implemented. The material models used in this study were selected from public articles. The ceramic material model is described by shock equation of state with Drucker-Prager strength model [6]. The aluminium 2017-T6 was chosen for backing plate which has Steinberg-Guinan strength model [3]. The epoxy resin which is widely used in ceramic and metal adhesive was set for bounding material and applied with Cowper-Symonds strength model [3]. Finally, the default data of tungsten material properties in software's library was selected for the projectile which also has Steinberg-Guinan strength model. Figure 2 depicts the finite element model of the ceramic/metal plate with the cylindrical projectile. The material damage was defined by the geometric strain limit and the material's maximum plastic strain.

As shown in Table 1, there are eight cases of simulation model in this study in order to investigate influences of tiles' geometry, boundary conditions and bounding materials. In order to define the tile size, the basic simulation was performed first. From the results of basic simulation supported by the data taken from Paul J. Hazell et al. [4], the appropriate ceramic tile size used for investigating the boundary effect is $30 \times 30 \times 9 \mathrm{~mm}$. for square tile and $15 \mathrm{~mm}$ (side length) $\times 9 \mathrm{~mm}$ for hexagonal tile. The aluminium plate has the same size as the ceramic tile but it has $6 \mathrm{~mm}$ thick. The solid cylinder with outside diameter $5.5 \mathrm{~mm}$ and $21 \mathrm{~mm}$ length was created to represent 7.62 AP Projectile's core. The thickness of epoxy is $2 \mathrm{~mm}$ around the tile.

\section{Results and Discussion}

The results from numerical simulation are illustrated in Table 2 and Figure 3. Figure $\mathbf{3}$ shows the stress plot when the projectile was stopped in each case. For fixed boundary constraint, the hexagonal tile can reduce the aluminium deformation compared with the square tile. This can be the result from the shorter distance from the center of the hexagonal tile to its boundary which gives the smaller tile's deflection. The model bounded by epoxy showed the lower protection performance as the deformation of the aluminium increased. This can be explained that the epoxy allowed small backward displacement of the ceramic tile after impact which caused more deformation on the aluminium plate. The study on the effect of the backing plate showed that small gap between the ceramic and aluminium increases the time to stop the projectile. Besides, it also reduces the strength of the ceramic tile as the maximum deformation increases when compared to the same boundary conditions. From the
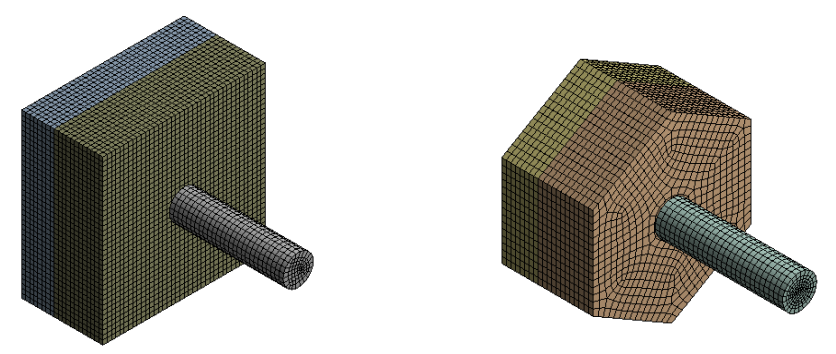

Figure 2. Finite element models of square tile and hexagonal tile.

Table 1. Numerical simulation boundary conditions.

\begin{tabular}{ccccc}
\hline Tile shape & Fixed boundary & Epoxy bound & Interface gap with fixed boundary & Interface gap with epoxy bound \\
\hline Square & Case 1 & Case 3 & Case 5 & Case 7 \\
Hexagonal & Case 2 & Case 4 & Case 6 & Case 8 \\
\hline
\end{tabular}




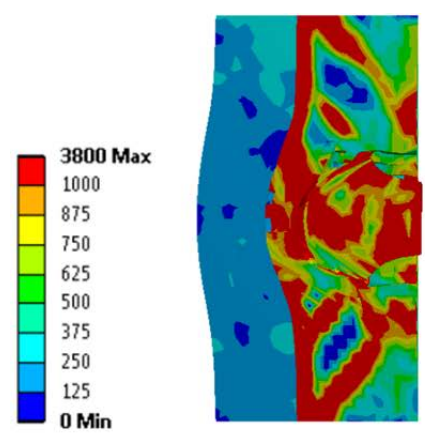

(a) Case 1

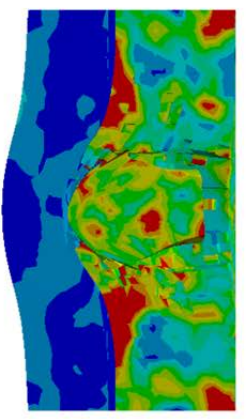

(e) Case 5

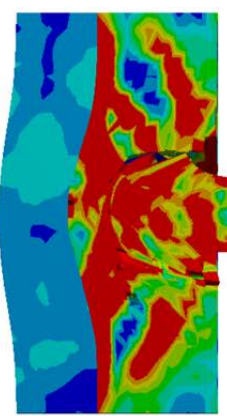

(b) Case 2

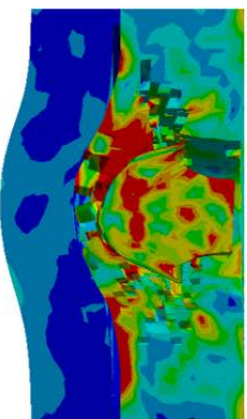

(f) Case6

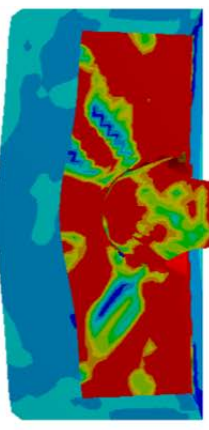

(c) Case3

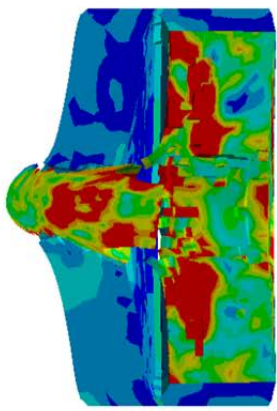

(g) Case7

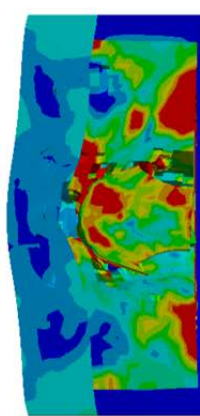

(d) Case4

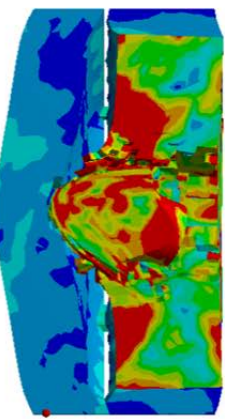

(h) Case8

Figure 3. Stress plot at the final states.

Table 2. Numerical results of different boundary conditions

\begin{tabular}{ccccccccc}
\hline & Case 1 & Case 2 & Case 3 & Case 4 & Case 5 & Case 6 & Case 7 & Case 8 \\
\hline Time when Projectile stopped $(\mu \mathrm{s})$ & 40 & 38 & 36 & 67.5 & 80 & 63 & - & 80 \\
Aluminium deformation $(\mathrm{mm})$ & 2.43 & 2.24 & 2.92 & 3.68 & 4.4 & 3.16 & - & 5.11 \\
Ceramic penetration & Yes & Yes & Yes & Yes & Yes & Yes & Yes & Yes \\
Steel penetration & No & No & No & No & No & No & Yes & No \\
\hline
\end{tabular}

animation results, the model with gap creates the crack on the ceramic tile faster than the model without gap. This is because the stress wave from the impact face reached the free surface behind the ceramic before the model with no gap and then the reflection wave was created and it caused the crack on the ceramic.

\section{Conclusion}

From this study, it shows that the boundary conditions affect the impact mechanism. Further study with smaller time step that can capture the impact behavior needs to be conducted for more accuracy. The different types of bounding material are also required for enhancing our understanding regarding the effects of different materials. In brief, the numerical simulation approach with element erosion setting is highly essential in explaining the damage behaviors of the ceramic.

\section{Acknowledgements}

The authors would like to present the appreciation to Defence Technology Institute (Public Organisation) for research facility and engineering information.

\section{References}

[1] Hazell, P.J., Appleby-Thomas, G.J. and Toone, S. (2014) Ballistic Compaction of a Confined Ceramic Powder by a 
Non-Deforming Projectile: Experiments and Simulations. Materials and Design, 56, 943-952. http://dx.doi.org/10.1016/j.matdes.2013.12.042

[2] Zeara, R., Sanchez-Saez, S., Perez-Castellanos, J.L. and Navarro, C. (2000) Modeling of the Adhesive Layer in Mixed Ceramic/Metal Armours Subjected to Impact. Composites: Part A, 31, 823-833. http://dx.doi.org/10.1016/S1359-835X(00)00027-0

[3] Lopez-Puente, J., Arias, A., Zaera, R. and Navarro, C. (2005) The Effect of the Thickness of the Adhesive Layer on the Ballistic Limit of Ceramic/Metal Armours. An Experimental and Numerical Study. International Journal of Impact Engineering, 32, 321-336. http://dx.doi.org/10.1016/j.ijimpeng.2005.07.014

[4] Hazell, P.J., Roberson, C.J. and Moutinho, M. (2008) The Design of Mosaic Armour: The Influence of Tile Size on Ballistic Performance. Materials and Design, 29, 1497-1503. http://dx.doi.org/10.1016/j.matdes.2008.03.003

[5] Carlucci, D.E. and Jacobson, S.S. (2007) Ballistics Theory and Design of Guns and Ammunition. CRC Press. http://dx.doi.org/10.1201/9781420066197

[6] (2003) Ballistic Impact on Ceramic/Aramid Armour System. AUTODYN. 